OPEN ACCESS

Edited by:

Jean-christophe Augustin, Ecole Nationale Vétérinaire d'Alfort,

France

Reviewed by:

Elliot Ryser,

Michigan State Police, United States

Antonio Valero,

Universidad de Córdoba, Spain

Helene Bergis,

Agence Nationale de Sécurité

Sanitaire de l'Alimentation,

de l'Environnement et du Travail

(ANSES), France

*Correspondence:

Mei-Jun Zhu

meijun.zhu@wsu.edu

Specialty section:

This article was submitted to

Food Microbiology,

a section of the journal

Frontiers in Microbiology

Received: 29 March 2017

Accepted: 10 July 2017

Published: 24 July 2017

Citation:

Sheng L, Edwards K, Tsai H-C, Hanrahan I and Zhu M-J (2017) Fate of Listeria monocytogenes on Fresh Apples under Different Storage Temperatures.

Front. Microbiol. 8:1396.

doi: 10.3389/fmicb.2017.01396

\section{Fate of Listeria monocytogenes on Fresh Apples under Different Storage Temperatures}

\author{
Lina Sheng ${ }^{1}$, Katheryn Edwards ${ }^{1}$, Hsieh-Chin Tsai ${ }^{1}$, Ines Hanrahan ${ }^{2}$ and Mei-Jun Zhu ${ }^{1 *}$ \\ ${ }^{1}$ School of Food Science, Washington State University, Pullman, WA, United States, ${ }^{2}$ Washington Tree Fruit Research \\ Commission, Wenatchee, WA, United States
}

Fresh apples are typically stored for up to 1 year commercially; different apple varieties require different storage temperatures to maintain their quality characteristics. There is sparse information available about Listeria monocytogenes survival on fresh apples under various storage temperatures. The objective of this study was to comprehensively evaluate the effect of storage temperature on apple fruit decay and L. monocytogenes survival. Unwaxed apple fruits of selected varieties (Fuji and Granny Smith) were dip inoculated in a three-strain L. monocytogenes cocktail to establish $\sim 3.5$ and $6.0 \log _{10}$ CFU/apple. Twenty-four hours post-inoculation, apples were subjected to $1,4,10$, or $22^{\circ} \mathrm{C}$ storage for up to 3 months. Apples under the different storage treatments were sampled at 1-, 4-, 7- and 14-day for short-term storage under all four tested temperatures, and 2-, 4-, 8-, and 12-week for long-term storage at 1, 4, and $10^{\circ} \mathrm{C}$. A set of uninoculated and unwaxed apples were simultaneously subjected to the previously mentioned storage temperatures and sampled biweekly for their total bacterial count (TPC) and yeasts/molds (Y/M) count. During the 2-week shortterm storage, L. monocytogenes population on organic Granny Smith apples stored at 1,4 , or $10^{\circ} \mathrm{C}$ was reduced by $0.2-0.3 \mathrm{Log}$. When apples were stored at $22^{\circ} \mathrm{C}$, there was a $0.5-1.2 \log _{10}$ CFU/apple reduction 14-day post storage dependent on the initial inoculation level. During the 12 -week cold storage under 1,4 , and $10^{\circ} \mathrm{C}$, L. monocytogenes count on organic Granny Smith apples decreased by 0.5-1.5 Log $\log _{10}$ CFU/apple for both inoculation levels. L. monocytogenes had similar survival pattern on conventional Granny Smith and Fuji apples with 0.8-2.0 $\log _{10}$ CFU/apple reduction over a 3-month cold storage period. Interestingly, both TPC and Y/M count were stable regardless of apple variety or cultivation practice during the 12-week storage at all tested temperatures. In summary, while L. monocytogenes did not proliferate on apple surfaces during 12 weeks of refrigerated storage, only a limited reduction of $L$. monocytogenes was observed in this study. Therefore, the apple industry cannot rely on cold storage alone to control this pathogen. Additional interventions are needed to eradicate Listeria on fresh apples during long-term cold storage.

Keywords: fresh apple, L. monocytogenes, survival, storage, fruit decay 


\section{INTRODUCTION}

Listeria monocytogenes is a major foodborne pathogen and causes about 1600 illnesses annually in the United States with a mortality rate of $\sim 16 \%$ (Scallan et al., 2011). L. monocytogenes is listed by the U.S. Food and Drug Administration as a "pathogen of concern" and has been singled out on readyto-eat (RTE) produce due to its nature as a environmental species, its frequent occurrence in many produce-associated environment and operations, and its high mortality rate (Scallan et al., 2011). Historically, L. monocytogenes outbreaks were associated with RTE meat such as turkey franks (CDC, 1989), hot dog (CDC, 1999), and delicatessen turkey meat (CDC, 2000). Dairy products, such as ice cream (Rietberg et al., 2016) and soft cheese products (Choi et al., 2014; Crowe et al., 2015; Heiman et al., 2016), are frequently involved in L. monocytogenes outbreaks. In recent years, increasing numbers of L. monocytogenes outbreaks were associated with fresh produce. The 2011 cantaloupe outbreak resulted in 143 hospitalizations and 33 deaths (Cosgrove et al., 2011). The 2014-2015 multistate L. monocytogenes outbreak related to caramel apples caused 35 illnesses in 12 states including 7 deaths (CDC, 2015). In addition, there were numerous L. monocytogenes recalls related to fresh apples (FDA, 2015a), sliced apples (CFIA, 2015; FDA, 2016), and stone fruits including whole peaches, nectarines, plums, and pluots (Jackson et al., 2015). The above evidence indicates $L$. monocytogenes has become an increasing threat to fresh produce safety and in particular to tree fruit producers.

Unique among other foodborne pathogens, L. monocytogenes has the ability to survive and even grow on fresh produce at refrigerated temperatures. L. monocytogenes was stable on lettuce during a 5-day storage at $5^{\circ} \mathrm{C}$ (Poimenidou et al., 2016) and tomatoes during the 20 -day storage under $10^{\circ} \mathrm{C}$ (Beuchat and Brackett, 1991). A recent study indicated that L. monocytogenes established on fresh Gala apples were stable during 15-day storage at $5^{\circ} \mathrm{C}$; while under the same conditions, the population of L. monocytogenes inoculated on caramel apples increased by $\sim 3 \log _{10}$ CFU/apple (Salazar et al., 2016). In addition, L. monocytogenes population increased by more than $3 \log _{10}$ CFU/apple in caramel apples with an inserted stick within 3 days of storage at $25^{\circ} \mathrm{C}$ (Glass et al., 2015; Salazar et al., 2016). These data have corroborated the concerns about L. monocytogenes and its negative effect on fresh produce safety. However, inadequate information is available about the behavior of L. monocytogenes on fresh apples. The Produce Rule of the Food Safety Modernization Act (FSMA) (FDA, 2015b) requires the fresh apple growers, packers, as well as processors to identify and adopt validated and effective preventive methods to reduce microbial risk in fresh apples by implementing worker training and hygienic practices, control of agricultural water, biological soil amendments, and wild animals, as well as sanitation of equipment, tools, and buildings. This study aimed to evaluate the fate of $L$. monocytogenes in fresh apples stored at different temperatures during both short- and longterm storage. Granny Smith apples (GSA) were studied since it's a cultivar involved in the L. monocytogenes outbreaks (FDA, 2014).
Both organic and conventional GSA were used to elucidate the potential influence of different native flora due to the cultivation differences (Camatti-Sartori et al., 2005). Conventional Fuji apples, which have a different surface structure, were also used in this study.

\section{MATERIALS AND METHODS}

\section{L. monocytogenes Strains and Culture Condition}

Listeria monocytogenes NRRL B-57618 (1/2a), NRRL B-33053 (4b), and NRRL-33466 (1/2b) were obtained from USDA-ARS culture collection [National Center for Agricultural Utilization Research (NRRL), Peoria, IL, United States] and maintained at $-80^{\circ} \mathrm{C}$ in Trypticase Soy Broth (Becton, Dickinson and Company, Sparks, MD, United States) supplemented with $0.6 \%$ Yeast Extract (Fisher Scientific, Fair Lawn, NJ, United States) (TSBYE) and 20\% (v/v) glycerol. The frozen culture was first activated in TSBYE at $37^{\circ} \mathrm{C}$ for $24 \mathrm{~h}$ statically, and then 1:500 subcultured in TSBYE for additional $24 \mathrm{~h}$ at $37^{\circ} \mathrm{C}$ before use.

\section{Inoculum Preparation}

Twice-activated bacteria cultures were centrifuged at 8,000 $\times g$ for $5 \mathrm{~min}$ at $4^{\circ} \mathrm{C}$. The resulting bacteria pellets were washed once and then re-suspended in Phosphate Buffered Saline (PBS, pH7.4). Equal volume of each L. monocytogenes strain was combined to make a three-strain cocktail and, subsequently, diluted to $\sim 6$ and $3 \log _{10} \mathrm{CFU} / \mathrm{ml}$ for apple inoculation. At the high inoculation level, there were $6.29 \pm 0.04$, $6.42 \pm 0.04$, and $6.01 \pm 0.07 \log _{10}$ CFU/apple on organic GSA, conventional GSA, and Fuji apples, respectively, immediately after inoculation. At the low inoculation level, $3.01 \pm 0.06$, $3.04 \pm 0.07$, and $3.48 \pm 0.03 \log _{10} \mathrm{CFU} /$ apple on organic GSA, conventional GSA, and Fuji apples, respectively, immediately after inoculation.

\section{Inoculation of Apples}

Whole fresh unwaxed GSA (organic and conventional) and Fuji apples (conventional) were donated by Allan Brothers Inc. and Stemilt Growers LLC. All fruit was of commercial maturity (the optimum maturity for the fresh market or storage) (Girschik et al., 2017). Apples were delivered from the commercial packing houses 1-week prior to inoculation and stored in a walk-in cooler $\left(4^{\circ} \mathrm{C}\right)$ temporarily. Medium size apples (with an average weight $\sim 220$ g) without cuts, bruising, or scars were selected and rinsed with cold tap water and dried on a bench top overnight to balance apple temperature to room temperature (RT). The apples were then inoculated with L. monocytogenes by submerging 15 apples each time in $5 \mathrm{~L}$ of three-strain L. monocytogenes cocktail inoculum solution at a selected inoculation level, and gently massaged by turning the apples in the inoculum for $10 \mathrm{~min}$ so that microorganisms were evenly distributed in the sample. For each variety at each inoculation level, four inocula were independently prepared with a total of $\sim 100$ apples inoculated per inoculum. Inoculated apples 


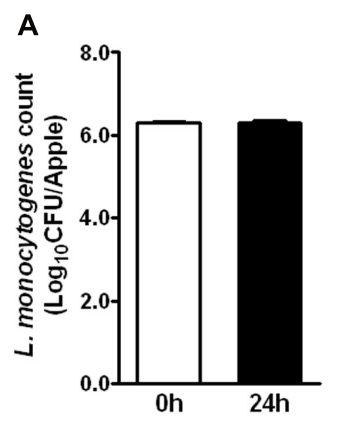

B

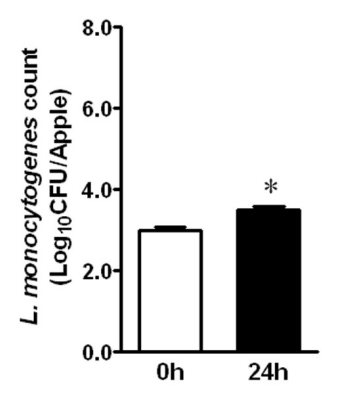

C

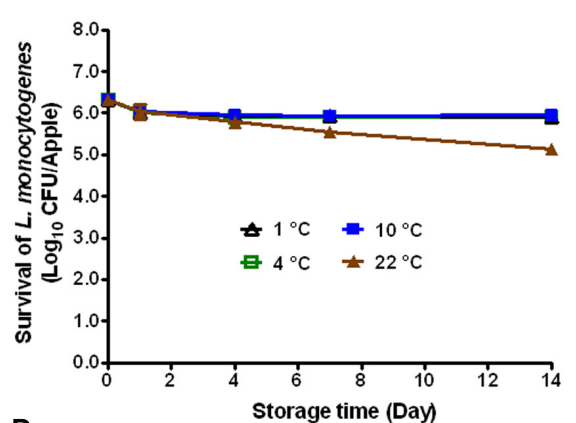

D

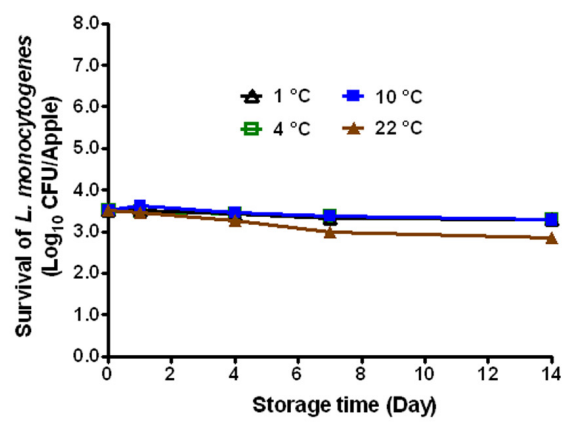

E

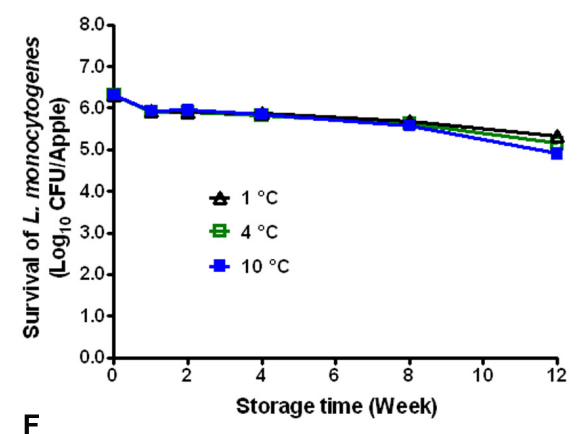

$\mathbf{F}$

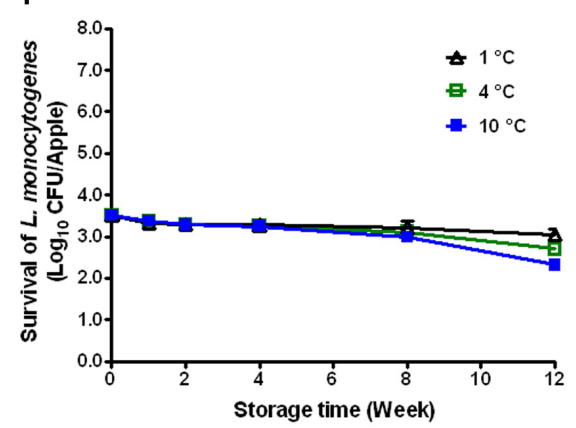

FIGURE 1 | Survival of Listeria monocytogenes on fresh organic Granny Smith apples during short- and long-term storage. (A,C,E) Inoculation level of $\sim 6$ Log 10 CFU/apple; (B,D,F) Inoculation level of $3.5 \log _{10}$ CFU/apple. Mean \pm SEM, $n=12$.

were dried for $24 \mathrm{~h}$ at RT $\left(\sim 22.5^{\circ} \mathrm{C}\right)$ before being subjected to storage. Twelve apples were sampled (three apples were randomly sampled from each of four inoculation batches) immediately following inoculation and $24 \mathrm{~h}$ post-inoculation for microbial enumeration to confirm inoculum uniformity, inoculation level, and the attachment of L. monocytogenes on fresh apples.

\section{Storage Treatment of Apples}

Both uninoculated and $24 \mathrm{~h}$ post-inoculation apples were placed in cardboard boxes and stored at $1^{\circ} \mathrm{C}, 4^{\circ} \mathrm{C}, 10^{\circ} \mathrm{C}$, and $\mathrm{RT}$ in lab-scale refrigerated incubators. Temperatures were monitored continuously by the built-in sensors, and double-checked weekly using two calibrated digital thermometers (Omega HH11, Omega Engineering Inc., Stamford, CT, United States) to ensure precision. Actual storage temperatures were $0.93 \pm 0.01$, $4.17 \pm 0.03,9.76 \pm 0.03$, and $22.65 \pm 0.03^{\circ} \mathrm{C}$. Twelve apples (three apples from each of the four inoculation batches) were sampled for microbial analysis from each temperature of each inoculation level (uninoculated, high and low inoculation levels) at selected sampling points. Uninoculated apples were sampled at 2-, 4-, 8-, 12-week of storage for total plate count (TPC) and yeasts/molds (Y/M) enumeration. Apples inoculated with L. monocytogenes were sampled at 1-, 4-, 7- and 14-day intervals for short-term storage, and at 2-, 4-, 8-, and 12-week for longterm cold storage $\left(1,4\right.$, and $\left.10^{\circ} \mathrm{C}\right)$.

\section{Microbial Analysis of Apples}

Each apple was placed into a sterile stomacher bag with $10 \mathrm{ml}$ sterile PBS and hand-rubbed for $1.5 \mathrm{~min}$. Wash solutions from each bag were 10-fold serially diluted with sterile PBS, and 0.1 or $1 \mathrm{ml}$ from appropriate dilutions was plated on duplicate appropriate plates. For inoculated apples, TSAYE (TSBYE with $1.5 \%$ agar) plates were used for apples with a L. monocytogenes level higher than $4.0 \log _{10} \mathrm{CFU} /$ apple where L. monocytogenes counts are much higher than the background flora $(<3.0$ $\log _{10}$ CFU/apple). TSAYE overlaid with Modified Oxford agar (MOX, Becton, Dickinson and Company, Sparks, MD, United States) plates were used for the enumeration of L. monocytogenes when its counts are lower than $4.0 \log _{10} \mathrm{CFU} /$ apple to discern from resident background flora of apples. The detection limit of L. monocytogenes is $10 \mathrm{CFU} /$ apple. For uninoculated apples, rub solutions were plated onto TSAYE for TPC and Potato Dextrose Agar (PDA, Becton, Dickinson and Company, Sparks, $\mathrm{MD}$, United States) plates for Y/M count. TSAYE plates were incubated at $35 \pm 1{ }^{\circ} \mathrm{C}$ for $48 \mathrm{~h}$. PDA plates were incubated at RT for 5 days.

\section{Statistical Analysis}

Data was analyzed by GLM from Statistical Analysis Systems (SAS, Cary, NC, United States). Mean values were compared by least significant difference (LSD) multiple-comparison test. $P$-values less than 0.05 were considered significant. Four batches of inocula were independently used to inoculate apples at each inoculation level for each variety. Each individual apple was an experimental unit. At each sampling point, three apples were randomly sampled from each batch $(N=12)$. Results were reported as mean \pm SEM (standard error of mean). 


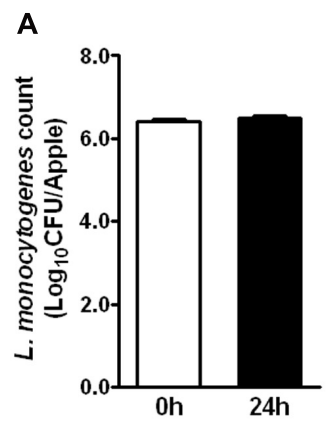

B

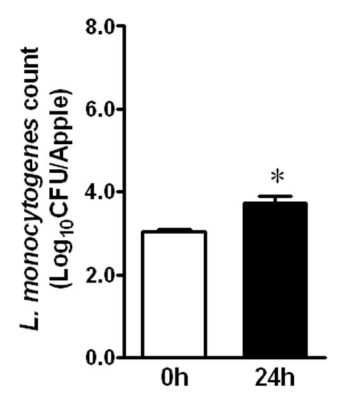

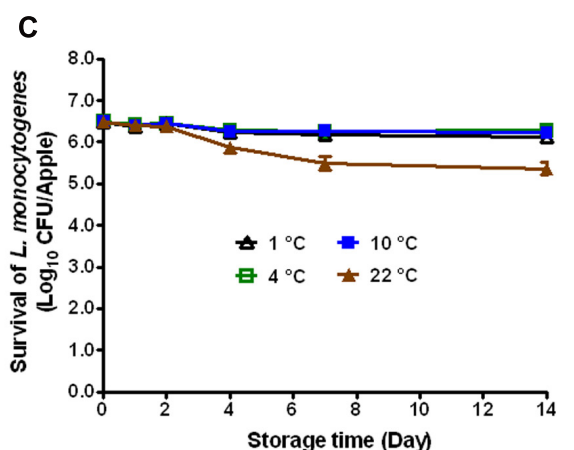

D

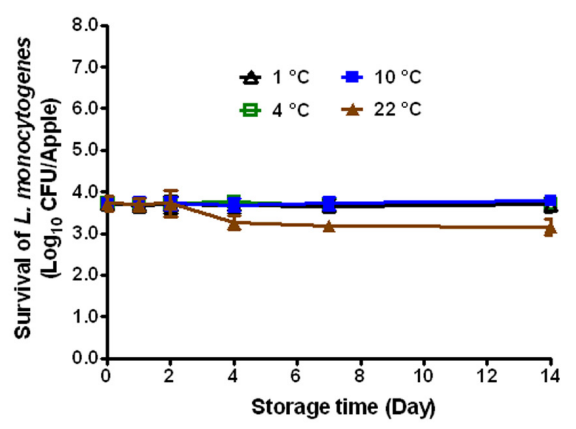

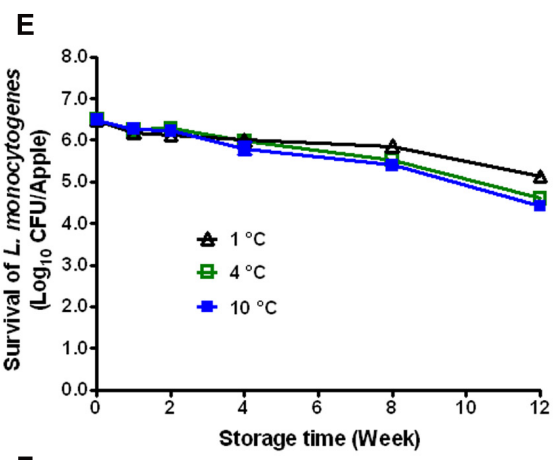

$\mathbf{F}$

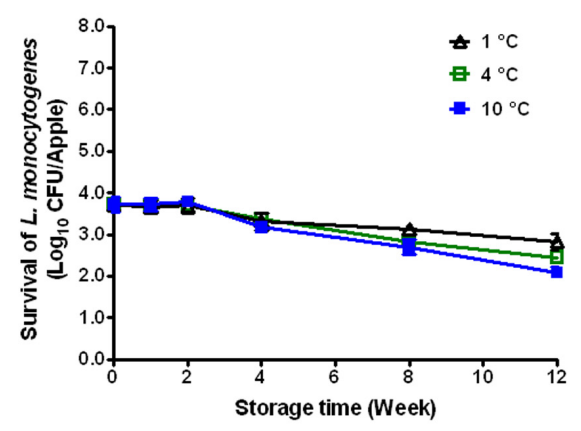

FIGURE 2 | Survival of L. monocytogenes on fresh conventional Granny Smith apples during short- and long-term storage. (A,C,E) Inoculation level of $\sim 6$ Log 10 CFU/apple; (B,D,F) Inoculation level of 3.5 Log $_{10}$ CFU/apple. Mean \pm SEM, $n=12$.

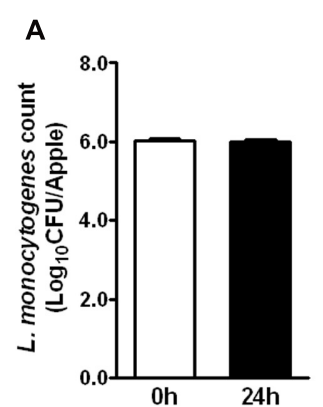

B

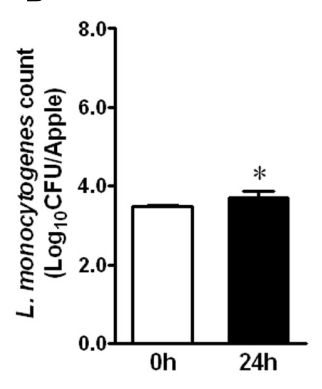

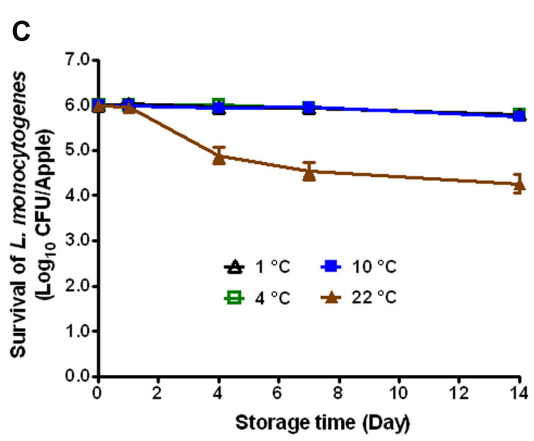

D

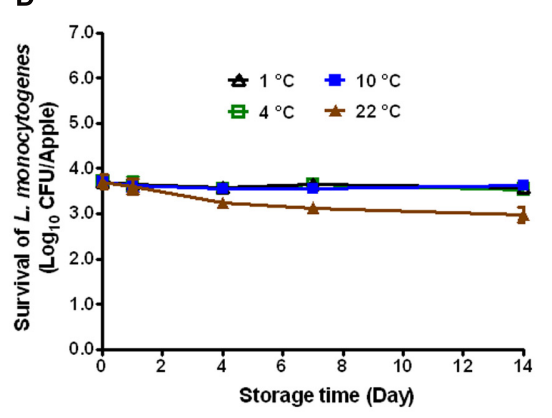

E

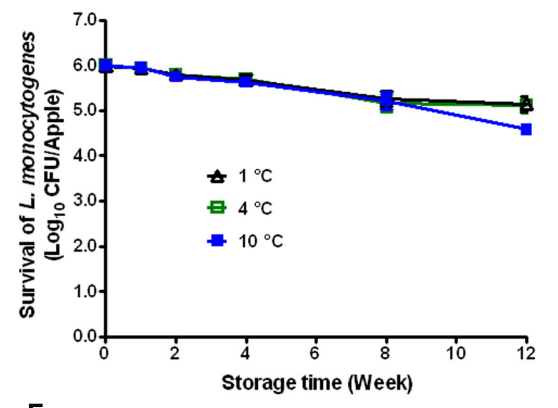

$\mathbf{F}$

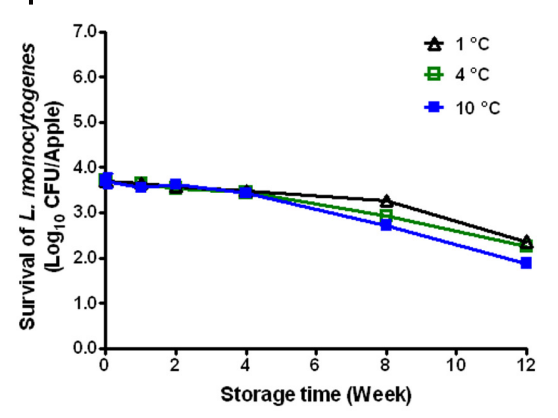

FIGURE 3 | Survival of L. monocytogenes on fresh conventional Fuji apples during short- and long-term storage. (A,C,E) Inoculation level of $\sim 6$ Log ${ }_{10}$ CFU/apple; (B,D,F) Inoculation level of 3.5 Log $_{10}$ CFU/apple. Mean \pm SEM, $n=12$. 


\section{RESULTS}

\section{L. monocytogenes Survival on Organic GSA}

Immediately after inoculation, organic GSA exhibited attachment of $6.29 \pm 0.04$ and $3.01 \pm 0.06 \log _{10}$ CFU/apple of L. monocytogenes, respectively (Figures $\mathbf{1 A}, \mathbf{B}$ ). After $24 \mathrm{~h}$ attachment at RT, bacterial counts were $6.31 \pm 0.06$ and $3.51 \pm 0.08$ for their respective inoculation levels (Figures 1A,B). There was a significant increase of $0.5 \log _{10}$ CFU/apple for the low inoculation level (Figure 1B, $P<0.05$ ). During 2 weeks of cold storage at 1,4 , and $10^{\circ} \mathrm{C}$, bacterial populations did not significantly change (Figures 1C,D). On apples stored at $22^{\circ} \mathrm{C}$, however, bacterial counts were reduced by 1.17 and $0.66 \log _{10}$ CFU/apple for the high and low inoculation levels, respectively (Figures 1C,D). L. monocytogenes established on fresh apples at either high or low level remained stable during cold storage. At both inoculation levels, only a $0.5-1.5 \log _{10}$ CFU/apple reduction on organic GSA during the 3-month cold storage was achieved (Figures 1E,F).

\section{L. monocytogenes Survival on Conventional GSA and Fuji Apples}

Apples from different agricultural practice or different varieties may behave differently in terms of bacterial adhesion and inactivation of the attached microorganisms to their surface. Therefore, we evaluated the fate of L. monocytogenes established on conventional GSA during storage. At the high inoculation level, L. monocytogenes maintained the same population size $24 \mathrm{~h}$ post-inoculation (Figure 2A). At the low inoculation level, the bacterial count was increased by $\sim 0.7 \log _{10}$ CFU/apple $24 \mathrm{~h}$ post-inoculation (Figure $\mathbf{2 B}, P<0.05$ ). After 2 weeks of cold storage, L. monocytogenes counts were slightly decreased for the high inoculation level, while no reduction was observed for the low inoculation level (Figures 2C,D). L. monocytogenes counts on apples stored at RT were reduced by 1.1 and 0.6 $\log _{10}$ CFU/apple for high and low inoculation level, respectively (Figures 2C,D). After 3-month cold storage, a 0.9-2.0 $\log _{10}$ CFU/apple reduction was achieved for both inoculation levels with $1^{\circ} \mathrm{C}$ showing the least reduction and $10^{\circ} \mathrm{C}$ the most (Figures 2E,F). Given the similar behavior of L. monocytogenes on organic and conventional GSA, the fate of L. monocytogenes was only accessed on conventional Fuji apples. Similar results were observed on inoculated conventional Fuji apples (Figure 3). After 2 weeks of cold storage, L. monocytogenes population on fresh Fuji apples was slightly reduced. There was about $\sim 1.7$ and $0.7 \log _{10} \mathrm{CFU} /$ apple reduction of $L$. monocytogenes on Fuji apples with high and low inoculation level, respectively, after 2-week of RT storage (Figures 3C,D), a slightly higher reduction than that on GSA. After 3-months, L. monocytogenes populations on Fuji apples were decreased by $0.8,0.9$, and 1.4 $\log _{10} \mathrm{CFU} /$ apple at 1,4 , and $10^{\circ} \mathrm{C}$, respectively, for the high inoculation level (Figure 3E). For the low inoculation level, L. monocytogenes counts on Fuji apples declined by 1.4, 1.5, and $1.8 \log _{10} \mathrm{CFU} /$ apple at 1,4 , and $10^{\circ} \mathrm{C}$, respectively (Figure 3F).

\section{Evolution of Spoilage Microbiota in GSA and Fuji Apples}

Bacteria, mold, and yeast can cause postharvest decay of apples (Janisiewicz and Korsten, 2002), reducing apple quality and causing a potential chemical (mycotoxin) hazard. Storage temperature has been reported to play a role in fruit decay (Mir et al., 2001). Therefore, TPC, in addition to $\mathrm{Y} / \mathrm{M}$ count was performed on uninoculated apples of the selected varieties stored under different temperatures. The initial TPC was $3.57 \pm 0.06$, $3.16 \pm 0.07$, and $3.55 \pm 0.06 \log _{10} \mathrm{CFU} /$ apple for organic GSA, conventional GSA, and conventional Fuji apples, respectively (Figures 4A-C). After 3-month storage, TPC was significantly reduced by $0.2-0.4 \log _{10}$ CFU/apple for organic GSA, and 0.5-0.6 $\log _{10}$ CFU/apple for conventional GSA and Fuji apples (Figures 4A-C). There was no difference in TPC on apples stored under different temperatures.

The initial Y/M count of organic GSA, conventional GSA, and conventional Fuji apples were $4.74 \pm 0.05,3.67 \pm 0.10$, and $4.73 \pm 0.07 \log _{10} \mathrm{CFU} /$ apple, respectively (Figures $4 \mathrm{D}-\mathrm{F}$ ). For both GSA (organic and conventional) and Fuji apples, Y/M count stayed the same throughout the cold storage with only a slight decline on apples stored at $22^{\circ} \mathrm{C}$ (Figures $4 \mathrm{D}-\mathrm{F}$ ).

\section{DISCUSSION}

\section{L. monocytogenes Attachment on Apples}

At the inoculation level of $\sim 6 \log _{10} \mathrm{CFU} /$ apple, $L$. monocytogenes population on fresh apples did not change during the $24 \mathrm{~h}$ establishment on the apple surface at RT, regardless of apple varieties or agricultural practice. Our results were corroborated with other previously published studies where the population of L. monocytogenes inoculated on whole strawberries at $\sim 7.5 \log _{10}$ CFU/berry was not significantly changed $24 \mathrm{~h}$ post-inoculation at $21^{\circ} \mathrm{C}$ (Flessa et al., 2005). Interestingly, at the low inoculation level, the population size of L. monocytogenes on apples slightly increased. Similarly, L. monocytogenes inoculated at $\sim 3 \log _{10}$ $\mathrm{CFU} / \mathrm{g}$ on whole tomatoes were increased by $\sim 1.5 \log _{10} \mathrm{CFU} / \mathrm{g}$ after 2-day storage at $21^{\circ} \mathrm{C}$ (Beuchat and Brackett, 1991). While the mechanism for the observed difference between high and low inoculation levels is unknown, it might be due to the competition for the limited nutrients on apple surface at the high inoculation level.

\section{L. monocytogenes Population on Fresh Apples Decreased during Room Temperature Storage}

A consumer survey study indicated that $42 \%$ of consumers have been reported to store apples at RT (Li-Cohen and Bruhn, 2002). Therefore, we evaluated behavior of $L$. monocytogenes on fresh apples during 2-week storage at RT. L. monocytogenes on apples of different cultivation practices (organic and conventional) or different varieties (GSA and Fuji apples) behaved similarly with 0.5-1.7 $\log _{10}$ CFU/apple reduction regardless of the inoculation level after 2-week storage under RT. Consistent with our findings, L. monocytogenes inoculated onto a GSA surface at $\sim 5 \log _{10}$ 

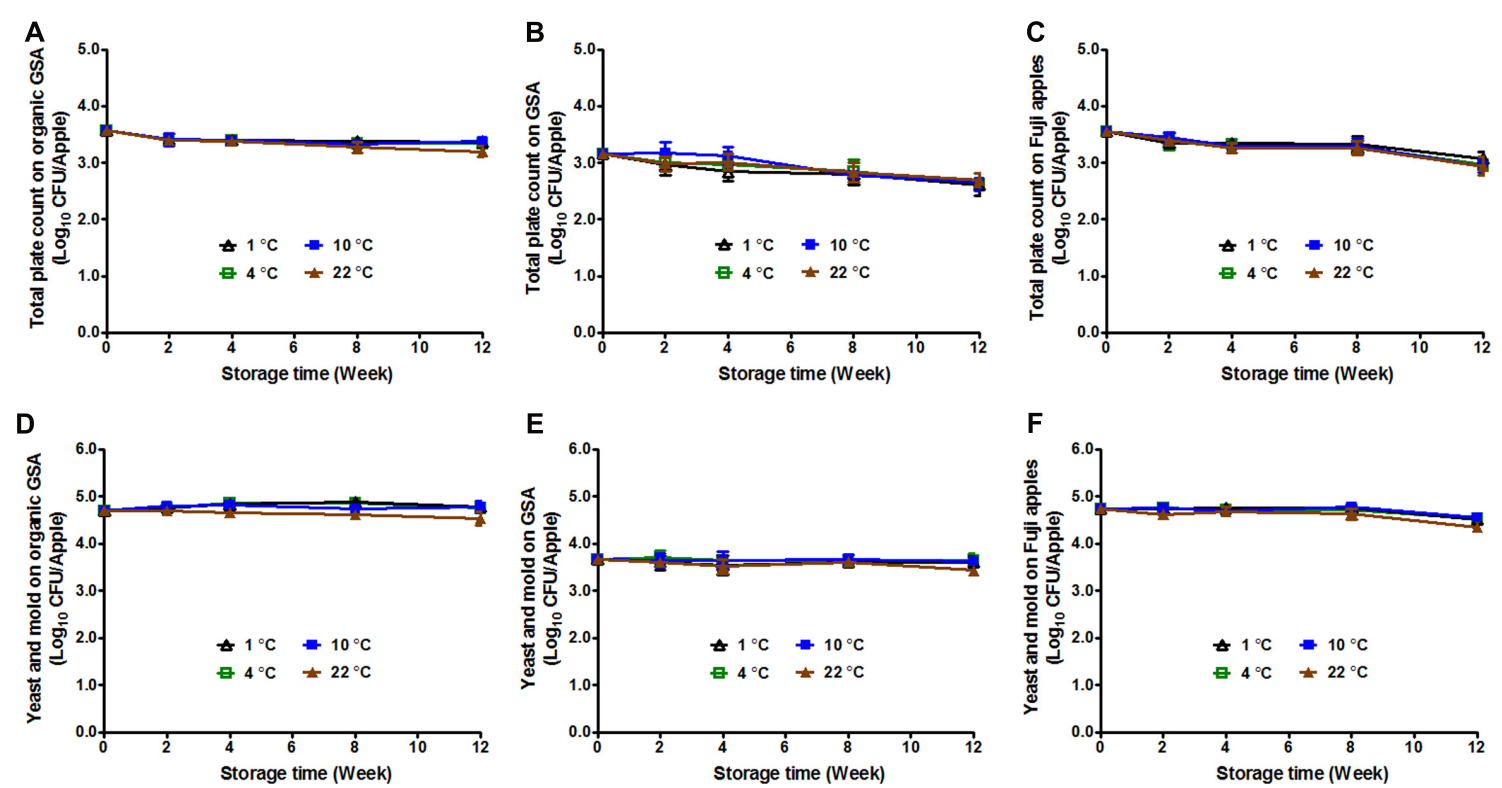

FIGURE 4 | Fruit decay during 3-month storage at different temperatures. Total plate count (A,B,C) and yeasts/molds count (D,E,F) were evaluated. (A,D) Organic Granny Smith apples; (B,E) Conventional Granny Smith apples; (C,F) Conventional Fuji apples. Mean \pm SEM, $n=12$.

CFU/apple was reduced by $\sim 1.5 \log _{10}$ CFU/apple after 15 day storage at $25^{\circ} \mathrm{C}$ (Salazar et al., 2016). The population of L. monocytogenes on whole strawberries at $7.1 \log _{10} \mathrm{CFU} /$ berry was declined by $1.0 \log _{10} \mathrm{CFU} /$ berry after 2-day storage under $24^{\circ} \mathrm{C}$ (Flessa et al., 2005). There was a $1.5 \log _{10} \mathrm{CFU} / \mathrm{cm}^{2}$ reduction of $L$. monocytogenes inoculated on whole cantaloupe surfaces at $\sim 3.5 \log _{10} \mathrm{CFU} / \mathrm{cm}^{2}$ during a 15 -day storage at $20^{\circ} \mathrm{C}$ (Ukuku and Fett, 2002).

\section{L. monocytogenes on Fresh Apples during Cold Storage}

Fresh apples are commercially stored at $\sim 1^{\circ} \mathrm{C}$ for extended periods of time (Mattheis et al., 1991). Apples are also held at $4^{\circ} \mathrm{C}$ in the packing house and consumer's refrigerators at home (Duvenage and Korsten, 2016). During the transportation, apples may be exposed to temperature abuse of $10^{\circ} \mathrm{C}$ (Huang et al., 2015). Therefore, all three temperatures were tested in this study for extended storage of 3 months. Our results showed that $L$. monocytogenes on apples was stable during the first 2 weeks at all three temperatures regardless of inoculation level, apple variety, or growing practice. A similar phenomenon was observed when L. monocytogenes (5 $\log _{10} \mathrm{CFU} / \mathrm{g}$ ) was inoculated onto whole Golden Delicious apples; there was no $L$. monocytogenes reduction during a 9-day storage at $4^{\circ} \mathrm{C}$ (Rodgers et al., 2004). Similarly, the population size of $L$. monocytogenes inoculated on whole tomatoes at $\sim 3.5 \log _{10} \mathrm{CFU} / \mathrm{g}$ remained at the same level throughout the 21 -day storage at $10^{\circ} \mathrm{C}$ (Beuchat and Brackett, 1991).

Survival of L. monocytogenes was similar among 1, 4, and $10^{\circ} \mathrm{C}$, which was higher than that of RT storage. It might because apples stored at RT produced more volatile compounds including those with antimicrobial effects such as alcohols, aldehydes, ketones, and esters compared with apples at refrigerated storage (HamiltonKemp et al., 1996; Matich et al., 1996; Dixon and Hewett, 2000), which facilitated L. monocytogenes death under RT storage. On molecular level, cold storage might increase survival through altering fatty acid composition of the bacterial membrane (Mastronicolis et al., 2005), which protects L. monocytogenes against other environmental stresses during storage (Lou and Yousef, 1997). Cold temperatures may have also induced the expression of general stress sigma factor $\sigma^{\mathrm{B}}$, facilitating L. monocytogenes survival (Hecker and Volker, 1998; Becker et al., 2000). However, L. monocytogenes inoculated on whole strawberries was reduced by $\sim 3 \log _{10}$ CFU/berry after 7 -day storage at $4^{\circ} \mathrm{C}$ (Flessa et al., 2005). These data indicate that a different food matrix and/or surface properties impacts L. monocytogenes survival at refrigerated temperature. The reduction of $L$. monocytogenes in strawberries might also be associated with the high moisture and low $\mathrm{pH}$ value of the fruit surface.

During 3 months of extended cold storage, reduction of L. monocytogenes on apples was temperature-dependent with relatively more reduction at $10^{\circ} \mathrm{C}$. In support of our data, less L. monocytogenes were recovered from stainless steel surfaces stored at $10^{\circ} \mathrm{C}$ than those at $4^{\circ} \mathrm{C}$ (Redfern and Verran, 2017). This might due to the induction of a more fierce stress response and accumulation of more low molecular weight solutes at lower temperatures (NicAogain and O'Byrne, 2016). The phenomenon could also resulted from temperature-dependent alteration in apple fruit quality during long-term storage (Mir et al., 2001) with more antimicrobial volatile compounds production at a higher cold storage temperature (Dixon and Hewett, 2000). Inoculation level, 
apple variety, or agricultural practice had minor influences on L. monocytogenes survival on apple surfaces during cold storage.

\section{Storage Temperatures Had No Effect on Apple Fruit Decay}

Resident bacteria, yeasts, and molds on apples contribute to fruit decay (Janisiewicz and Korsten, 2002). Thus, their fate was further studied over storage. TPC and Y/M counts stayed at the similar level throughout the 3-month storage under different temperatures for apples of the selected varieties and practices. In support of our finding, mesophilic aerobic microorganism, and $\mathrm{Y} / \mathrm{M}$ counts were not changed on raw carrots during 18day storage at $5^{\circ} \mathrm{C}$ (Beuchat and Brackett, 1990). However, inconsistent with our results, TPC and Y/M counts of raw carrots were increased by $\sim 0.5$ and $1.5 \log _{10} \mathrm{CFU} / \mathrm{g}$, respectively, after the 7-day storage at $15^{\circ} \mathrm{C}$ (Beuchat and Brackett, 1990). In another study, TPC as well as Y/M counts on whole tomatoes increased during the 20-day storage at $10^{\circ} \mathrm{C}$ and the 8-day storage at $21^{\circ} \mathrm{C}$ (Beuchat and Brackett, 1991). The difference in background flora behavior might due to different crop surfaces. The low-moisture condition on apple surface in this study might prevent the growth of resident bacteria, yeasts, and molds.

\section{CONCLUSION}

Short-term RT storage resulted in 0.5-1.7 $\log _{10}$ CFU/apple reduction of $L$. monocytogenes on fresh apples. Growers and

\section{REFERENCES}

Becker, L. A., Evans, S. N., Hutkins, R. W., and Benson, A. K. (2000). Role of sigma(B) in adaptation of Listeria monocytogenes to growth at low temperature. J. Bacteriol. 182, 7083-7087. doi: 10.1128/Jb.182.24.7083-7087.2000

Beuchat, L. R., and Brackett, R. E. (1990). Inhibitory effects of raw carrots on Listeria monocytogenes. Appl. Environ. Microbiol. 56, 1734-1742.

Beuchat, L. R., and Brackett, R. E. (1991). Behavior of Listeria monocytogenes inoculated into raw tomatoes and processed tomato products. Appl. Environ. Microbiol. 57, 1367-1371.

Camatti-Sartori, V., da Silva-Ribeiro, R. T., Valdebenito-Sanhueza, R. M., Pagnocca, F. C., Echeverrigaray, S., and Azevedo, J. L. (2005). Endophytic yeasts and filamentous fungi associated with southern Brazilian apple (Malus domestica) orchards subjected to conventional, integrated or organic cultivation. J. Basic Microbiol. 45, 397-402. doi: 10.1002/jobm.200410547

CDC (1989). Epidemiologic notes and reports: listeriosis associated with consumption of turkey franks. Morb. Mortal. Wkly Rep. 47, 267-268.

CDC (1999). Update: multi-state outbreak of listeriosis-United States, 1998-1999. Morb. Mortal. Wkly. Rep. 47, 1117-1118.

CDC (2000). Multistate outbreak of listeriosis-United States, 2000. Morb. Mortal. Wkly. Rep. 49, 1129-1130.

CDC (2015). Multistate Outbreak of Listeriosis Linked to Commercially Produced, Prepackaged Caramel Apples Made from Bidart Bros. Available at: http://www. cdc.gov/listeria/outbreaks/caramel-apples-12-14/

CFIA (2015). Food Recall Warning - Sliced Apples and Products Containing Sliced Apples Recalled due to Listeria monocytogenes. Available at: http://www.inspection.gc.ca/about-the-cfia/newsroom/food-recall-warnings/ complete-listing/2015-04-30/eng/1430431517170/1430431518530

Choi, M. J., Jackson, K. A., Medus, C., Beal, J., Rigdon, C. E., Cloyd, T. C., et al. (2014). Notes from the field: multistate outbreak of listeriosis linked to packers in the apple industry often assume that $L$. monocytogenes on apples will die off during long-term cold storage. However, a limited reduction of L. monocytogenes on apple surfaces occurred during 12 weeks of refrigerated storage regardless of inoculation levels, apple varieties, or agricultural practices. This indicates that cold storage alone is not enough for eradicating $L$. monocytogenes from fresh apple surfaces. Additional intervention steps or hurdles for removal of L. monocytogenes are urgently needed during fruit storage or thereafter, such as during the packing of the fruit, in the apple industry.

\section{AUTHOR CONTRIBUTIONS}

LS is the main person who performed the experiment and wrote the manuscript. KE helped with the experiment and manuscript revision. H-CT helped with the experiment. IH provided the experimental apples and helped revise the manuscript. M-JZ guided the experimental design, experiment, as well as the manuscript revision.

\section{ACKNOWLEDGMENTS}

This activity was partially funded by the Washington Tree Fruit Research Commission. We thank Allan Brothers Inc. and Stemilt Growers LLC for their generous donation of fresh apples. We thank Xiaofei Sun, Tonia Green, Yansong Xue, Michael H. Taylor, and Xia Song for their help in preparation of inoculated apples and microbial analysis.

soft-ripened cheese-United States, 2013. MMWR. Morb. Mort. Wkly. Rep. 63, 294-295.

Cosgrove, S., Cronquist, A., Wright, G., Ghosh, T., Vogt, R., Teitell, P., et al. (2011). Multistate outbreak of listeriosis associated with Jensen Farms cantaloupe-United States, August-September 2011 (Reprinted from MMWR, vol 60, pg 1357-1358, 2011). Jama J. Am. Med. Assoc. 306, 2321-2321.

Crowe, S. J., Mahon, B. E., Vieira, A. R., and Gould, L. H. (2015). Vital signs: multistate foodborne outbreaks - United States, 2010-2014. Morb. Mort. Wkly. Rep. 64, 1221-1225. doi: 10.15585/mmwr.mm6443a4

Dixon, J., and Hewett, E. W. (2000). Factors affecting apple aroma/flavour volatile concentration: a review. N. Z. J. Crop Hortic. Sci. 28, 155-173. doi: 10.1080/ 01140671.2000 .9514136

Duvenage, S., and Korsten, L. (2016). Effect of temperature and nutrient concentration on survival of foodborne pathogens in deciduous fruit processing environments for effective hygiene management. J. Food Prot. 79, 1959-1964. doi: 10.4315/0362-028X.JFP-16-050

FDA (2014). FDA Investigates Listeria monocytogenes Illnesses Linked to Caramel Apples. Available at: http://www.fda.gov/Food/RecallsOutbreaksEmergencies/ Outbreaks/ucm 427573.htm

FDA (2015a). Del Monte Fresh Produce N.A. Inc., Recalls Limited Quantity of Fresh Apples due to Possible Health Risk. Available at: http://www.fda.gov/Safety/ Recalls/ucm467078.htm

FDA (2015b). FSMA Final Rule on Produce Safety. Available at: http://www.fda.gov/ Food/GuidanceRegulation/FSMA/ucm334114.htm

FDA (2016). Fresh from Texas Recalls Apple Product because of Possible Health Risk. Available at: https://www.fda.gov/Safety/Recalls/ucm494345.htm

Flessa, S., Lusk, D. M., and Harris, L. J. (2005). Survival of Listeria monocytogenes on fresh and frozen strawberries. Int. J. Food Microbiol. 101, 255-262. doi: 10.1016/j.ijfoodmicro.2004.11.010 
Girschik, L., Jones, J. E., Kerslake, F. L., Robertson, M., Dambergs, R. G., and Swarts, N. D. (2017). Apple variety and maturity profiling of base ciders using UV spectroscopy. Food Chem. 228, 323-329. doi: 10.1016/j.foodchem.2017. 02.012

Glass, K. A., Golden, M. C., Wanless, B. J., Bedale, W., and Czuprynski, C. (2015). Growth of Listeria monocytogenes within a Caramel-Coated apple microenvironment. mBio 6:e01232-15. doi: 10.1128/mBio.01232-15

HamiltonKemp, T. R., Archbold, D. D., Loughrin, J. H., Collins, R. W., and Byers, M. E. (1996). Metabolism of natural volatile compounds by strawberry fruit. J. Agric. Food Chem. 44, 2802-2805. doi: 10.1021/Jf9601980

Hecker, M., and Volker, U. (1998). Non-specific, general and multiple stress resistance of growth-restricted Bacillus subtilis cells by the expression of the sigmaB regulon. Mol. Microbiol. 29, 1129-1136. doi: 10.1046/j.1365-2958.1998. 00977.x

Heiman, K. E., Garalde, V. B., Gronostaj, M., Jackson, K. A., Beam, S., Joseph, L., et al. (2016). Multistate outbreak of listeriosis caused by imported cheese and evidence of cross-contamination of other cheeses, USA, 2012. Epidemiol. Infect. 144, 2698-2708. doi: 10.1017/S095026881500117X

Huang, J., Luo, Y., and Nou, X. (2015). Growth of Salmonella enterica and Listeria monocytogenes on fresh-cut cantaloupe under different temperature abuse scenarios. J. Food Prot. 78, 1125-1131. doi: 10.4315/0362-028X.JFP-14- 468

Jackson, B. R., Salter, M., Tarr, C., Conrad, A., Harvey, E., Steinbock, L., et al. (2015). Notes from the field: listeriosis associated with stone fruit-United States, 2014. Morb. Mort. Wkly. Rep. 64, 282-283.

Janisiewicz, W. J., and Korsten, L. (2002). Biological control of postharvest diseases of fruits. Annu. Rev. Phytopathol. 40, 411-441. doi: 10.1146/annurev.phyto.40. 120401.130158

Li-Cohen, A. E., and Bruhn, C. M. (2002). Safety of consumer handling of fresh produce from the time of purchase to the plate: a comprehensive consumer survey. J. Food Prot. 65, 1287-1296. doi: 10.4315/0362-028X-65.8.1287

Lou, Y., and Yousef, A. E. (1997). Adaptation to sublethal environmental stresses protects Listeria monocytogenes against lethal preservation factors. Appl. Environ. Microbiol. 63, 1252-1255.

Mastronicolis, S. K., Arvanitis, N., Karaliota, A., Litos, C., Stavroulakis, G., Moustaka, H., et al. (2005). Cold dependence of fatty acid profile of different lipid structures of Listeria monocytogenes. Food Microbiol. 22, 213-219. doi: 10.1016/j.fm.2004.08.002

Matich, A. J., Rowan, D. D., and Banks, N. H. (1996). Solid phase microextraction for quantitative headspace sampling of apple volatiles. Anal. Chem. 68, 4114-4118. doi: 10.1021/Ac9604548

Mattheis, J. P., Buchanan, D. A., and Fellman, J. K. (1991). Change in apple fruit volatiles after storage in atmospheres inducing anaerobic metabolism. J. Agric. Food Chem. 39, 1602-1605. doi: 10.1021/Jf00009a012
Mir, N. A., Curell, E., Khan, N., Whitaker, M., and Beaudry, R. M. (2001). Harvest maturity, storage temperature, and 1-MCP application frequency alter firmness retention and chlorophyll fluorescence of 'Red chief Delicious' apples. J. Am. Soc. Hortic. Sci. 126, 618-624.

NicAogain, K., and O'Byrne, C. P. (2016). The role of stress and stress adaptations in determining the fate of the bacterial pathogen Listeria monocytogenes in the food chain. Front. Microbiol. 7:1865. doi: 10.3389/Fmicb.2016.01865

Poimenidou, S. V., Chatzithoma, D. N., Nychas, G. J., and Skandamis, P. N. (2016). Adaptive response of Listeria monocytogenes to heat, salinity and low $\mathrm{pH}$, after habituation on cherry tomatoes and lettuce leaves. PLoS ONE 11:e0165746. doi: 10.1371/journal.pone.0165746

Redfern, J., and Verran, J. (2017). Effect of humidity and temperature on the survival of Listeria monocytogenes on surfaces. Lett. Appl. Microbiol. 64, 276-282. doi: 10.1111/lam.12714

Rietberg, K., Lloyd, J., Melius, B., Wyman, P., Treadwell, R., Olson, G., et al. (2016). Outbreak of Listeria monocytogenes infections linked to a pasteurized ice cream product served to hospitalized patients. Epidemiol. Infect. 144, 2728-2731. doi: 10.1017/S0950268815003039

Rodgers, S. L., Cash, J. N., Siddiq, M., and Ryser, E. T. (2004). A comparison of different chemical sanitizers for inactivating Escherichia coli O157:H7 and Listeria monocytogenes in solution and on apples, lettuce, strawberries, and cantaloupe. J. Food Prot. 67, 721-731. doi: 10.4315/0362-028X-67.4.721

Salazar, J. K., Carstens, C. K., Bathija, V. M., Narula, S. S., Parish, M., and Tortorello, M. L. (2016). Fate of Listeria monocytogenes in fresh apples and caramel apples. J. Food Prot. 79, 696-702. doi: 10.4315/0362-028X.JFP-15-442

Scallan, E., Hoekstra, R. M., Angulo, F. J., Tauxe, R. V., Widdowson, M. A., Roy, S. L., et al. (2011). Foodborne illness acquired in the United States-major pathogens. Emerg. Infect. Dis. 17, 7-15. doi: 10.3201/eid1701.091101p1

Ukuku, D. O., and Fett, W. (2002). Behavior of Listeria monocytogenes inoculated on cantaloupe surfaces and efficacy of washing treatments to reduce transfer from rind to fresh-cut pieces. J. Food Prot. 65, 924-930. doi: 10.4315/0362028X-65.6.924

Conflict of Interest Statement: The authors declare that the research was conducted in the absence of any commercial or financial relationships that could be construed as a potential conflict of interest.

Copyright (C) 2017 Sheng, Edwards, Tsai, Hanrahan and Zhu. This is an open-access article distributed under the terms of the Creative Commons Attribution License (CC BY). The use, distribution or reproduction in other forums is permitted, provided the original author(s) or licensor are credited and that the original publication in this journal is cited, in accordance with accepted academic practice. No use, distribution or reproduction is permitted which does not comply with these terms. 